\title{
In vitro and in vivo evaluation of nano-based films for buccal delivery of zolpidem
}

\section{Bandar Essa AL-DHUBIAB(a)}

(a)King Faisal University, College of Clinical Pharmacy, Department of Pharmaceutical Sciences, Al-Ahsa, Saudi Arabia
Declaration of Interests: The authors certify that they have no commercial or associative interest that represents a conflict of interest in connection with the manuscript.

Corresponding Author:

Bandar E. Al-Dhubiab

E-mail: baldhubiab@kfu.edu.sa

DOI: 10.1590/1807-3107BOR-2016.vol30.0126

Submitted: Dec 19, 2015

Accepted for publication: Apr 5, 2016

Last revision: Sep 22,2016
Abstract: Insomnia is becoming increasingly prevalent in the world general population. Therapies used by patients include over-the-counter therapies, herbal and dietary supplements, and pharmacological or nonpharmacological treatments. Among these, zolpidem is a pharmacological treatment popularly used for insomnia. Zolpidem is well tolerated and especially efficacious for initiation of sleep, and therefore is effective for the treatment of sleep-onset insomnia. The purpose of the present study was to design and evaluate zolpidem nanoparticle-impregnated buccal films to prolong the duration of its action. Zolpidem nanospheres were prepared by double emulsion solvent evaporation and then loaded into buccoadhesive films (Z1-Z4) comprised of different concentrations of HPMC K100, Eudragit ${ }^{\circledR}$ RL 100, and carbopol 974P. The prepared films were characterized for physicomechanical properties, mucoadhesion, percent hydration, in vitro drug release, ex vivo permeation, and in vivo studies. In vitro drug release was found to depend upon film composition. Ex vivo studies showed that film Z4 had the highest flux. In vivo studies revealed that administration of zolpidem nanosphere-impregnated film enhanced absorption of the drug $(p<0.0001)$, with a higher peak plasma concentration $(52.54 \pm 8.22 \mathrm{ng} / \mathrm{mL})$ and area under the curve from time 0 to a $(236.00 \pm 39.51 \mathrm{ng} . \mathrm{h} / \mathrm{mL})$ than oral administration. The increase in time taken to reach the maximum drug concentration $(1.5 \mathrm{~h})$ further signifies the potential of these films to provide prolonged drug release. Given these promising results, we concluded that these buccal films could be an alternative route for effective zolpidem delivery.

Keywords: Nanospheres; Zolpidem; Sleep Initiation and Maintenance Disorders; Polymers.

\section{Introduction}

Insomnia is becoming increasingly rampant, affecting from $10 \%$ to around $60 \%$ of the general population. ${ }^{1}$ It is characterized by one or more of the following subjective complaints: a) a sleep onset latency of over $30 \mathrm{~min}, \mathrm{~b}$ ) nightly sleep duration of less than $6 \mathrm{~h}$, and/or c) waking too early and being unable to return to sleep (also referred to as unrefreshed sleep). ${ }^{2}$ Studies conducted in the general population indicate that adults experience more of these symptoms, while the National Sleep Foundation's Sleep in America Poll 2005 found that more than 50\% of Americans were 
afflicted by one or more of the above symptoms at least a few nights each week. A very startling revelation of the poll was that in the previous year, at least one-third of the population had experienced one or more of the above symptoms.

Insomnia is not a stand-alone problem; it leads to multiple daytime consequences that can impact the lives of patients. ${ }^{3}$ Individuals suffering from insomnia are prone to emotional distress and frequent health problems. ${ }^{4,5}$ Even $1-2 \mathrm{~h}$ of sleep deprivation may impair alertness, concentration, attention, cognitive abilities, mood, memory, and pain threshold. ${ }^{6,7}$ Consequently, patients often use nonprescription therapies, like over-the-counter sedatives, herbal, and/or dietary supplements, to address symptoms, and a number of pharmacological and nonpharmacological therapies have been found to be valuable for the treatment of insomnia. Pharmacological treatments include the use of hypnotics. Unfortunately, first-generation hypnotics (barbiturates, carbamates, chloral hydrate, and methaqualone) typically exert a prolonged hypnosedative effect, which impacts patient work performance the following day. Benzodiazepine hypnotics were introduced in the 1970s and were a major breakthrough in the treatment of insomnia. Compared to first-generation hypnotics, benzodiazepine hypnotics show improved safety in terms of tolerance and withdrawal effects. However, side effects, such as hangover, dependency, and rebound insomnia, can occur with declining use. ${ }^{8}$ Thus, benzodiazepines still fall short of being ideal compounds for promoting physiological sleep.

With the introduction of cyclopyrrolones (zopiclone), imidazopyridines (zolpidem), and pyrazolopyrimidines (zaleplon) for insomnia, a further decline in benzodiazepine use has been observed world-wide. Among these new compounds, zolpidem tartrate salt has become the most popular treatment for insomnia. ${ }^{2}$ Zolpidem is an effective hypnosedative similar to benzodiazepines, with minimal muscle relaxant, anxiolytic, and anticonvulsant properties. Compared to benzodiazepines, zolpidem has a more selective $y$-aminobutyric acid receptor-binding profile. Zolpidem has an approximately 10 -fold higher affinity to $\mathrm{a} 1$-containing $\gamma$-aminobutyric acid receptors than for those containing a2- or a3-subunits.
It is this binding profile that differentiates zolpidem from benzodiazepines and also explains why it has relatively little myorelaxant or anticonvulsant activity and low potential for dependence, abuse, and/or withdrawal symptoms. ${ }^{9}$ Minor side effects of zolpidem are partly due to the relatively short half-life of this drug in the circulation. ${ }^{10}$

Immediate-release forms of zolpidem have a rapid onset of action, and the duration of action is limited due to its short life. As such, these dosage forms have limited usefulness for maintaining sleep through the night and have led to the development of extended-release formulations. ${ }^{11,12}$ Working along similar lines, we propose an alternative drug delivery route involving a buccoadhesive film impregnated with zolpidem nanospheres that can provide a rapid onset of action together with prolonged release from poly(lactic-co-glycolic acid) [PLGA] nanospheres. These films have the potential to help patients with sleep onset, as well as maintain longer sleep intervals.

\section{Methodology}

\section{Chemicals}

The zolpidem was provided by Ind-Swift Laboratories (Punjab, India). PLGA (50:50; Mw 7,000-17,000 dalton), Hydroxypropyl methyl cellulose (HPMC) K100, propylene glycol, methanol, ethyl alcohol, dichloromethane, and polyvinyl alcohol were purchased from Sigma Aldrich (St. Louis, MO, USA). Eudragit ${ }^{\circledast}$ RL 100 (Evonik, Darmstadt, Germany), polyvinyl pyrrolidone K-30, and dibutyl phthalate (Loba Chemie, Mumbai, India) were purchased commercially. Carbopol 974P, hydroxypropyl methyl cellulose, and ethyl cellulose were provided by Ind-Swift, Ltd. (Baddi, Himachal Pradesh, India). All other chemicals and reagents used in the study were of analytical grade.

\section{Analytical method}

High-performance liquid chromatography (HPLC; Model 600 pump with UV-Vis detector; Waters, Milford, MA) was utilized for quantitative analysis of zolpidem in samples. A reverse-phase Symmetry C18 analytical column $(15 \times 4.6 \mathrm{~mm}, 5 \mu \mathrm{m}$ particles; Waters) with a precolumn was used for elution; 
samples $(20 \mu \mathrm{L})$ were injected and analyzed at $245 \mathrm{~nm}$. The mobile phase consisted of a methanol-deionized water mixture (75:25), and a flow rate of $0.8 \mathrm{~mL} / \mathrm{min}$. A calibration curve was prepared at $245 \mathrm{~nm}$ using methanol as the solvent, and linearity was obtained. The zolpidem concentration contained within the nanospheres was determined by dissolving a known amount of nanospheres in $10 \mathrm{~mL}$ of dichloromethane, ${ }^{13}$ vortexing for $1 \mathrm{~min}$, and filtering before HPLC analysis. The results obtained were expressed as encapsulation efficiency, expressed as (recovered mmol zolpidem/g nanospheres)/(loaded mmol zolpidem/g polymer) ${ }^{*} 100$.

\section{Preparation of nanospheres}

A double emulsion-solvent evaporation method was used for preparation of zolpidem-loaded PLGA nanospheres. ${ }^{14}$ Briefly, PLGA $(100 \mathrm{mg})$ was added to dichloromethane while stirring until a clear solution formed. Solutions $(1 \mathrm{~mL})$ containing $2,4,6,8$, or $10 \mathrm{mg} / \mathrm{mL}$ zolpidem were added slowly to the polymeric solution with high-speed stirring to form the primary emulsion. The primary emulsion was then added to $25 \mathrm{~mL}$ of polyvinyl alcohol solution [2\% (w/v)] and homogenized using a high-speed homogenizer for $2 \mathrm{~min}$. This secondary emulsion mixture was stirred for $12 \mathrm{~h}$ at room temperature to remove residual solvent. The resultant dispersion was centrifuged at 20,000 rpm for $30 \mathrm{~min}$ and the supernatant was removed. The prepared nanospheres were washed thrice with water to remove polymer residue and then lyophilized.

\section{Particle size characterization}

The lyophilized nanospheres were suspended in deionized water. Particle size, particle size distribution, and Zeta potential measurements were carried out using a Nano-ZS Zetasizer (Malvern, Westborough, MA, USA).

\section{Preparation of zolpidem-loaded buccoadhesive films}

Zolpidem-loaded buccal films were prepared as outlined in Table 1. Eudragit ${ }^{\circledR}$ RL 100 was added to an appropriate quantity of ethyl alcohol [70\% (v/v)] and stirred using a magnetic stirrer. HPMC K100
Table 1. Composition of different polymeric buccal films containing zolpidem nanospheres (Z1-Z4).

\begin{tabular}{lcccc}
\hline $\begin{array}{l}\text { Formulation } \\
\text { code }\end{array}$ & $\begin{array}{c}\text { Zolpidem } \\
(\mathrm{mg})^{*}\end{array}$ & $\begin{array}{c}\text { HPMC K100 } / \mathrm{v}) \\
(\%\end{array}$ & $\begin{array}{c}\text { Eudragit RL } \\
100(\% \mathrm{w} / \mathrm{v})\end{array}$ & $\begin{array}{c}\text { Carbopol } \\
974 \mathrm{P}(\% \mathrm{w} / \mathrm{v})\end{array}$ \\
\hline Z1 & 1 & 1.0 & 15.0 & 3.0 \\
Z2 & 1 & 2.0 & 12.5 & 3.0 \\
Z3 & 1 & 3.0 & 10.0 & 3.0 \\
Z4 & 1 & 4.0 & 7.5 & 3.0 \\
\hline
\end{tabular}

*Amount of nanospheres equivalent to $1 \mathrm{mg}$ zolpidem $/ \mathrm{cm}^{2}$.

was mixed with a suitable quantity of deionized water containing propylene glycol $[2 \%(\mathrm{v} / \mathrm{v})]$ using a mechanical stirrer. Carbopol 974P was separately dispersed in deionized water with stirring. HPMC K100 and carbopol 974P dispersions were mixed together and then mixed with a Eudragit ${ }^{\circledR}$ RL 100 dispersion. At this stage, an appropriate quantity of drug-loaded nanospheres was incorporated into the above mixture to form a single uniform dispersion. This dispersion was sonicated to remove any entrapped air bubbles. The amount of nanospheres incorporated into the films was such that each $\mathrm{cm}^{2}$ of film contained $1 \mathrm{mg}$ of zolpidem. An accurately measured volume of the final dispersion was casted onto a custom-made glass mold (area, $6 \mathrm{~cm}^{2}$ ). The mold was covered using an inverted glass funnel designed to allow slow evaporation of the film at $35{ }^{\circ} \mathrm{C}$ for $24 \mathrm{~h}$.

A backing membrane was prepared for the mucoadhesive films using ethyl cellulose. A $5 \%(\mathrm{w} / \mathrm{v})$ solution of ethyl cellulose in ethanol [95\% (v/v)] was prepared and mixed with dibutyl phthalate [20\% $(\mathrm{v} / \mathrm{v})]$ as a plasticizer and casted on the glass mold. These films were left to dry at room temperature for $24 \mathrm{~h}$. The dried backing membranes were attached to the buccoadhesive films using a $5 \%(\mathrm{w} / \mathrm{v})$ solution of polyvinyl pyrrolidone K-30. The final films consisted of mucoadhesive adhered to the backing membrane. These films were cut to $1 \mathrm{~cm}^{2}$ sizes and stored in a desiccator until use.

\section{Physicochemical characterization of buccal films}

The physical characteristics of the prepared films (i.e., color, softness, transparency, thickness, and peelability) were evaluated. The color and 
transparency were noted against a white background and light, respectively; softness and peelability were noted by touch. Film thickness was noted using a screw gauge (Mitutoyo) at five different locations; a $1 \mathrm{~cm}^{2}$ film size was used for measuring the $\mathrm{pH}$ of the film. The film was allowed to swell in $5 \mathrm{~mL}$ of distilled water for $20 \mathrm{~min}$ and then removed to drain before measuring the $\mathrm{pH}$ of the film using a flat surface electrode (Orion 4 Star Benchtop, ThermoFisher Scientific, Waltham, MA, USA).

\section{Drug content}

Buccal films $\left(1 \mathrm{~cm}^{2}\right)$ were punched from different regions of larger pieces and soaked in $50 \mathrm{~mL}$ of an ethanol-water mixture (50:50) with stirring. The extraction period was determined to be $12 \mathrm{~h}$ since the next sampling period at $24 \mathrm{~h}$ did not improve extraction of zolpidem. The solution was then centrifuged at $10,000 \mathrm{rpm}$ for $2 \mathrm{~min}$, then the supernatant was filtered and analyzed by HPLC. ${ }^{15}$

\section{Percent swelling}

Swelling studies were carried out for the prepared films by evaluating their percent hydration. Films samples $\left(1 \mathrm{~cm}^{2}\right)$ were weighed and placed on a stainless steel mesh ${ }^{16}$ and then immersed in phosphate buffered saline at $37 \pm 1^{\circ} \mathrm{C}$. The mesh was removed from the buffer at predetermined time points and wiped with tissue paper; the weight of the wiped film was noted. The percent hydration of the films was determined by the equation below: ${ }^{17}$

$\%$ hydration $=\left[\left(\mathrm{W}_{2}-\mathrm{W}_{1}\right) / \mathrm{W}_{2}\right] \times 100$,

where $W_{1}$ is the initial weight of the film, and $W_{2}$ is the weight of the film after swelling.

\section{Drug release}

In vitro release studies of zolpidem from the buccal films were completed using a paddle-over-disc USP XXIV Type II apparatus (Electrolab TDC 50, India). Film samples $(2 \times 1 \mathrm{~cm})$ were cut and pasted on a glass slide using a double-sided adhesive tape. The glass slide with the buccal film was immersed in $900 \mathrm{~mL}$ of simulated saliva ( $\mathrm{pH}$ 6.2) maintained at $37 \pm 1^{\circ} \mathrm{C}$; the paddle was rotated at $50 \mathrm{rpm}$. Samples of dissolution medium were withdrawn at predetermined time intervals, filtered, and analyzed by HPLC.

\section{Mucoadhesive strength}

The mucoadhesive strength of the film was measured using a texture analyzer (Stable Micro Systems, Ltd., Surrey, UK). Rabbit cheek pouch epithelium was used as the biological substrate and was mounted on the stationary platform of the texture analyzer. ${ }^{18}$ The assembly with cheek mucosal epithelium was filled with $2 \mathrm{~mL}$ of buffer solution to keep the tissue moist. A piece of the film $\left(1 \mathrm{~cm}^{2}\right)$ was cut and adhered to the probe of the texture analyzer using cyanoacrylate adhesive. The probe of the texture analyzer was lowered to make a contact with the mucosa. The epithelium was kept in contact with the film for $1 \mathrm{~min}$, and the following parameters were used: pretest speed, $0.5 \mathrm{~mm} / \mathrm{s}$; applied force, $1 \mathrm{~N}$; post-test probe speed, $0.5 \mathrm{~mm} / \mathrm{s}$. The maximum amount of force required to pull the film from the rabbit mucosal epithelium was taken as the mucoadhesive strength.

\section{Ex vivo permeation studies}

A Franz diffusion cell was used to carry out permeation studies. ${ }^{19}$ Rabbit buccal mucosa was used as the permeation medium for zolpidem. Freshly prepared rabbit buccal mucosa with the smooth surface facing the donor compartment was mounted between the donor and receptor compartment of the Franz diffusion cell. Zolpidem-loaded (nanospheres) buccal films measuring $0.6 \mathrm{~cm}^{2}$ were punched using a biopsy punch and adhered onto the rabbit mucosa on the donor compartment. Simulated saliva $(5 \mathrm{~mL})$ filled the receptor compartment, and then the donor and receptor compartments of the cell were clamped together. The diffusion cell was maintained at $37 \pm 0.2{ }^{\circ} \mathrm{C}$ and the diffusion medium was stirred at $50 \mathrm{rpm}$. At a predetermined time point, a $1 \mathrm{~mL}$ sample of the dissolution medium was withdrawn and replaced using fresh simulated saliva maintained at $37 \pm 0.2^{\circ} \mathrm{C}$. Withdrawn samples were filtered and analyzed by HPLC.

\section{Scanning electron microscopy}

Scanning electron microscopy (SEM) was utilized for observing buccal film samples. Samples of buccal film for SEM were mounted with silver electrical tape and coated with gold in a neutral environment 
under reduced pressure (SCD005 Baltek Sputter Coater). Samples were visualized with varying magnifications using a Joel 457-V SEM (Tokyo, Japan) and photographed. ${ }^{20}$

\section{In vivo study}

Male white rabbits $(2.25-2.75 \mathrm{~kg})$ housed in an animal housing facility were used for in vivo studies. The animals were maintained on a $12 \mathrm{~h}$ light/12h dark cycle and provided access to food and water ad libitum. Animals used for the study were fasted overnight with free access to water then anesthetized for 4-5 h using intramuscular injection of ketamine and/or xylazine. ${ }^{21}$ Buccal film samples $\left(1 \mathrm{~cm}^{2}\right)$ were slightly wetted using $30 \mu \mathrm{L}$ of water and applied to the buccal mucosa of the rabbits. Control animals were given an oral solution of zolpidem equivalent to a $1 \mathrm{mg}$ dose. At predetermined time points, blood samples $(500 \mu \mathrm{L})$ were withdrawn from the marginal ear vein using a needle. These samples were mixed with an equal amount of acetonitrile and centrifuged at 10,000 rpm for 10 minutes. The supernatant was filtered and analyzed by HPLC.

\section{Data analysis}

The cumulative amount of zolpidem that permeated across the buccal surface was plotted against time. The steady-state flux was estimated from the slope of the linear portion of the plot. ${ }^{22}$ An unpaired Student's t-test was carried out using GraphPad Prism 5.0 (San Diego, CA, USA); a p-value of $<0.05$ was considered statistically significant. Mean values \pm standard errors were calculated using values from six trials.

\section{Results and Discussion}

Nanocarriers as drug delivery systems have the ability to improve the overall pharmacological and therapeutic efficacy of drug molecules. This, together with the availability of a plethora of polymers, has contributed to efficient, safe, and successful delivery of a number of therapeutic agents. Nanocarriers possess the ability to provide drug release at a controlled rate and enhance bioavailability and adhesion to biological membranes. ${ }^{23}$ A number of buccal films impregnated with nanospheres have been developed and shown to enhance the therapeutic efficacy of drugs, improve pharmacokinetic profiles, and modulate drug release. ${ }^{24,25}$ Additionally, entrapment of the drug in a polymeric carrier tends to increase their stability.

Biodegradable polymeric carriers have their own set of advantages. PLGA is a biodegradable and biocompatible polymer approved by the US Food and Drug Administration that is useful for delivery of different drugs, particularly hydrophobic/hydrophilic molecules. Not only does it have the ability to protect drug molecules, it also provides prolonged drug release.${ }^{14}$ Consequently, we chose PLGA for delivery of zolpidem nanospheres in the current study, and the entrapment efficiency of the nanospheres was found to be $65 \%-72 \%$.

Particle size, distribution, and zeta potential were measured using a Zetasizer. Particle size of the prepared nanospheres was found to range from 100 to $350 \mathrm{~nm}$ (average, $200 \mathrm{~nm}$ ). The average zeta potential and polydispersity index were $\sim 32.07 \mathrm{mV}$ and 0.17 , respectively. Buccal films were formulated making use of these nanospheres as outlined in Table 1. Two hydrophilic mucoadhesive polymers, carbopol 974P and HPMC K100, were used for preparation of the film. The water-insoluble, film-forming polymer Eudragit ${ }^{\circledR}$ RL 100 was added to induce film formation and provide greater mechanical strength to the film for a longer period of time. Previous studies have shown the combination of carbopol 974P and HPMC K100 provides adequate swelling and mucoadhesive strength to the film. ${ }^{15,18,26}$ Different combinations of these three polymers were mixed to form films of varying composition (named Z1-Z4). Previous literature notes that these polymers are capable of controlling the release of pharmaceutically active compounds. ${ }^{14,18}$ The film composition (i.e., amount of hydrophilic/hydrophobic polymer) was varied to optimize the drug release profile as outlined in Table 1. The amount of carbopol 974P [3\% (w/v)] and the concentration of the plasticizer propylene glycol $[2 \%(\mathrm{w} / \mathrm{v})]$ used for film formation were kept constant.

Prepared films were off-white, soft, translucent, homogenous, and peelable. Film thickness is an indicator of dose accuracy and bioadhesion; ${ }^{16}$ the thickness of the film was found to range from 287 to $342 \mathrm{~nm}$ for Z1-Z4 (Table 2). Also, the thickness of 
each of film was uniform in all batches of a particular composition. The measured thickness of prepared films was ideal for application on buccal mucosa and for bioadhesion. ${ }^{11} \mathrm{pH}$ measurements revealed that all of the prepared films had a comparable buccal $\mathrm{pH}$ (6.82-7.21), which will not cause any irritation to buccal mucosa after application. ${ }^{18}$

Measurement of drug content is important to ensure the presence of the drug in the film, as well as its uniform distribution. ${ }^{16,18}$ Film samples $\left(1 \mathrm{~cm}^{2}\right)$ were punched from different regions of larger films and analyzed. The results showed the zolpidem content ranged from $94.12 \%$ to $98.14 \%$ of the theoretical value, with a low standard deviation $(< \pm 3.45 \%)$ in all cases. These results indicate the prepared films had a uniform drug distribution within.

Assessment of swelling capacity is important to understanding the bioadhesive properties of the film and drug release rate. Swelling of film depends on its structure, as well as the nature of its polymer matrix. ${ }^{24,25}$ As the polymeric film comes in contact with water, water molecules diffuse into the polymeric matrix, thereby hydrating the film. Hydration causes the film to swell, which facilitates diffusion of the drug from the film. As hydration continues, swelling increases, which leads to separation of nanospheres from the film. ${ }^{14}$ As indicated in Figure 1, all of the prepared films showed rapid hydration in the initial $2 \mathrm{~h}$ of the study, which is in agreement with several earlier studies. ${ }^{14,18,25}$ This was followed by a relatively slower phase of hydration from 2 to 8 hours. Percent hydration at $2 \mathrm{~h}$ was found to be $29.65 \% \pm 2.56 \%, 33.98 \% \pm 3.67 \%$, $43.45 \% \pm 6.33 \%$, and $54.56 \% \pm 5.23 \%$ in Z1-Z4 film, respectively. A higher initial hydration is suggestive of a higher hydration potential and good adhesion

Table 2. Evaluation of thickness, $\mathrm{pH}$ and drug content of different buccal films (mean $\pm S D, n=6$ ).

\begin{tabular}{lccc}
\hline $\begin{array}{l}\text { Formulation } \\
\text { code }\end{array}$ & $\begin{array}{c}\text { Thickness } \\
(\mathrm{nm})\end{array}$ & $\mathrm{pH}$ & $\begin{array}{c}\text { Percentage drug } \\
\text { content/cm }\end{array}$ \\
\hline Z1 & $342 \pm 28$ & $7.21 \pm 0.53$ & $94.12 \pm 2.18$ \\
Z2 & $328 \pm 20$ & $7.11 \pm 0.32$ & $94.42 \pm 3.45$ \\
Z3 & $307 \pm 18$ & $6.82 \pm 0.27$ & $97.36 \pm 3.13$ \\
Z4 & $287 \pm 19$ & $7.14 \pm 0.24$ & $98.14 \pm 2.96$ \\
\hline
\end{tabular}

with the buccal mucosa. The different films (Z1-Z4) showed different hydration characteristics since they were comprised of different combinations of hydrophilic (HPMC K100 and carbopol 974P) and hydrophobic (Eudragit RL 100) polymers. As the content of hydrophilic polymer in the film increased, water uptake and swelling of the film increased, which improved the percent hydration; the percent hydration was highest for film Z4, followed by Z3, $Z 2$, and Z1. This observation is in agreement with data reported by Kumria et al. ${ }^{27}$ All of the prepared films showed adequate hydration and provided good bioadhesion.

The prepared films were impregnated with nanospheres, and as a prerequisite, the nanospheres must be released from the film into the buccal mucosa. Release of nanospheres from the film is governed by the separation and diffusion of nanospheres from the polymer matrix. ${ }^{23}$ Once the nanospheres enter the buccal mucosa, the drug must be released in order to be absorbed into the circulation. Drug release from the matrix is governed by the solubility of the drug, diffusion of the drug from the nanosphere matrix, and erosion of the matrix. To study this behavior of drug release, in vitro dissolution studies were conducted. Figure 2 depicts the cumulative percent drug versus time profile of the nanosphere-impregnated buccoadhesive films; all of the prepared films showed a distinct drug release profile. In film Z1, release of zolpidem was found to be slower than from film Z2,

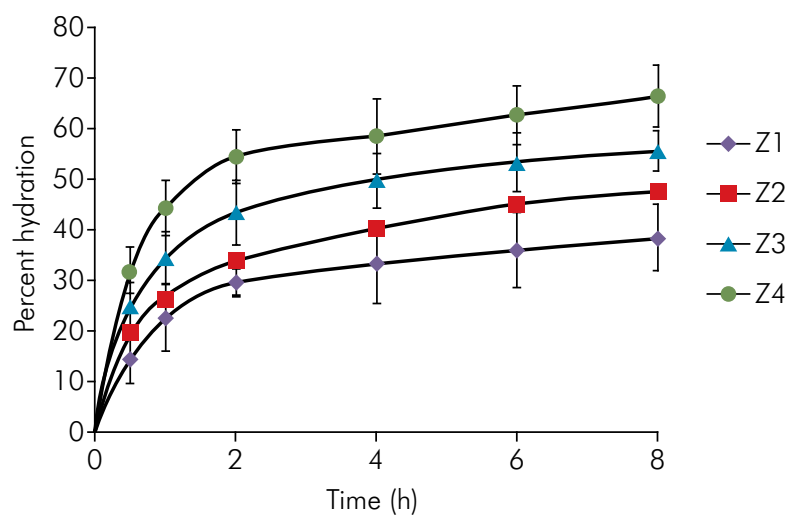

Figure 1. Percent hydration of prepared buccal films over $8 \mathrm{~h}$ determined using $1 \mathrm{~cm}^{2}$ pieces of film. Values represent the mean of six trials \pm standard deviation. 
which, in turn, showed slower release than Z3 and Z4. Film Z3 showed a cumulative percent drug release of $35.23 \% \pm 5.21 \%, 57.64 \% \pm 6.23 \%, 79.60 \% \pm 6.34 \%$, and $97.60 \% \pm 6.32 \%$ in $2,4,6$, and $8 \mathrm{~h}$, respectively. After $8 \mathrm{~h}$, drug release from film Z1 and Z2 was $82.98 \% \pm 5.32 \%$ and $88.61 \% \pm 6.71 \%$, while film Z4 showed nearly complete drug release $(99.10 \% \pm 3.02 \%)$. The difference in drug release from the different films could be attributed to their difference in polymeric composition. Several groups have reported similar observations in drug release when polymer composition was varied..$^{24,25,28}$ At the end of our study, films Z1 and Z2 showed the slowest drug release, and, therefore, were excluded from further studies.

Adequate mucoadhesion is a primary requisite for films to successfully deliver drugs to the buccal epithelium. Measurement of mucoadhesive strength allows assessment of the binding capability and retention characteristics of the film to the buccal mucosa. ${ }^{16}$ The mucoadhesive strengths of films Z3 and $\mathrm{Z} 4$ measured using rabbit mucosa as the substrate were found to be 7.89 and $8.34 \mathrm{~N}$, respectively. Based on evidence from the literature, ${ }^{24,25}$ these films likely possess adequate buccoadhesive strength to withstand the pressure exerted by movement in the buccal cavity. Mucoadhesive strength values of Z3 and Z4

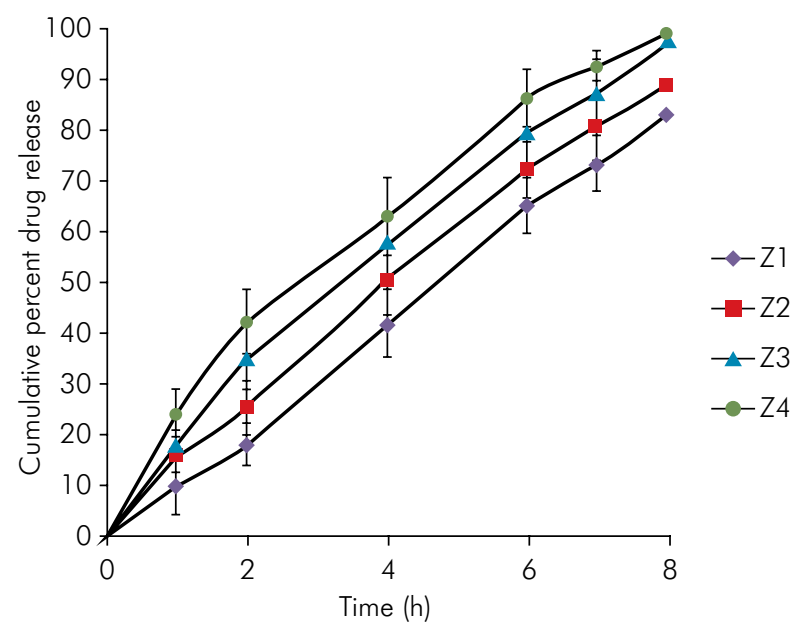

Figure 2. Comparison of the cumulative percentage of zolpidem released from different buccal films at various time points. In vitro drug release tests were carried out by placing film samples $(2 \times 1 \mathrm{~cm})$ in a USP apparatus (paddle-over-disc) using simulated saliva as a dissolution medium. Values represent the mean of six trials \pm standard deviation. were statistically significant $(p=0.0023)$, signifying that their film compositions had an effect on their mucoadhesive strength. Films consisted of two mucoadhesive polymers, HPMC K100 and carbopol 974P, and a film-forming polymer, Eudragit ${ }^{\circledR}$ RL 100. As the content of mucoadhesive polymer(s) increased, the film's mucoadhesion increased. These findings are in agreement with earlier reports, suggesting an increase in HPMC K100 content in the film increases its mucoadhesive strength. ${ }^{27}$

The absorption kinetics of drugs across biological membranes was studied using ex vivo permeation studies. ${ }^{29}$ Permeation across these membranes is a function of the physicochemical properties of the drug molecules and type of biological membrane used. ${ }^{30,31}$ Herein, rabbit buccal mucosa was used to simulate human buccal membranes. Ex vivo permeation studies were conducted for films Z3 and Z4 to assess the permeation of zolpidem across the membrane (Figure 3). We found permeation was higher for film $\mathrm{Z} 4$ compared to $\mathrm{Z} 3$, and flux values for $\mathrm{Z3}$ and $\mathrm{Z} 4$ were $75.39 \pm 12.53$ and $93.87 \pm 17.43 \mu \mathrm{g} / \mathrm{cm}^{2} / \mathrm{h}$, respectively. The cumulative amount of drug permeated at the end of $6 \mathrm{~h}$ was $452.39 \pm 23.22$ and $563.22 \pm 46.45 \mu \mathrm{g} / \mathrm{cm}^{2}$ for $\mathrm{Z3}$ and Z4, respectively. Drug-loaded nanospheres were found to permeate across the membrane in the first hour of the current permeation study, similar to other research groups. ${ }^{24,25}$ This shows the potential

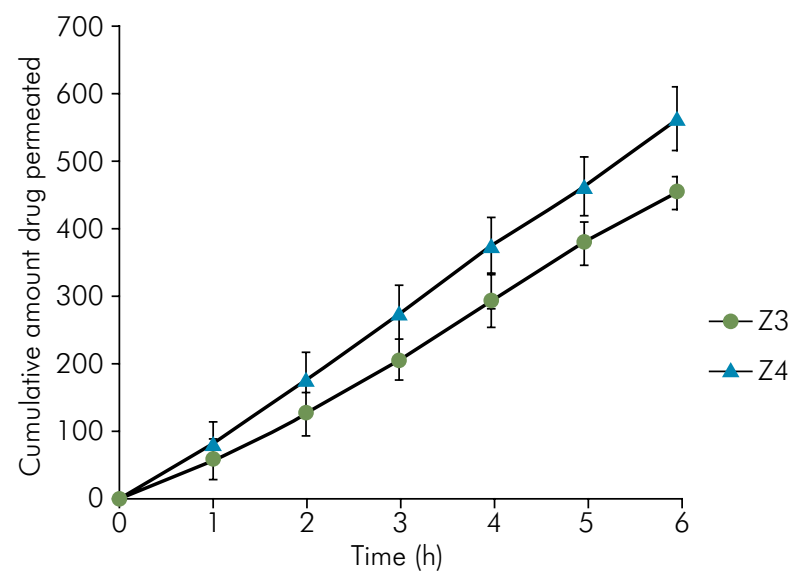

Figure 3. Comparison of ex vivo permeation profile of zolpidem from selected buccal films (Z3 and Z4) using rabbit buccal mucosa over $6 \mathrm{~h}$ in a Franz diffusion cell. Values represent the mean of six trials \pm standard deviation. 
of these nanospheres to rapidly permeate into and through the buccal mucosa. Because film Z4 showed a higher flux, it was selected for further studies.

SEM was used to obtain a high resolution 3-dimensional photograph of zolpidem nanosphere-impregnated buccal films. Indeed, the SEM images showed topographical and morphological features of the nanospheres incorporated into the buccal film. ${ }^{14}$ As shown in Figure 4, the nanospheres were found to be smooth and uniform. The film formed was also smooth and had all the desired characteristics ideal for buccal use.

To understand drug absorption characteristics and parameters that influence it, in vivo studies were carried out on rabbits ${ }^{16}$ in order to understand the actual pharmacokinetics. Film Z4 was selected for in vivo study, and the pharmacokinetics of zolpidem nanosphere-impregnated buccal films was compared with oral delivery of zolpidem. Rabbits have reportedly been the first choice of animal model for carrying out drug studies such as this one. ${ }^{16,32}$ Buccoadhesive films were applied to animals for $4 \mathrm{~h}$ and a similar dose was used for oral administration. The dose of zolpidem (1 mg) was calculated using an equation described by Nair and Jacob. ${ }^{33}$

Different pharmacokinetic parameters, such as the area under the curve from time 0 to $a$, the peak plasma concentration (Cmax), and time taken to reach the maximum drug concentration (Tmax), were

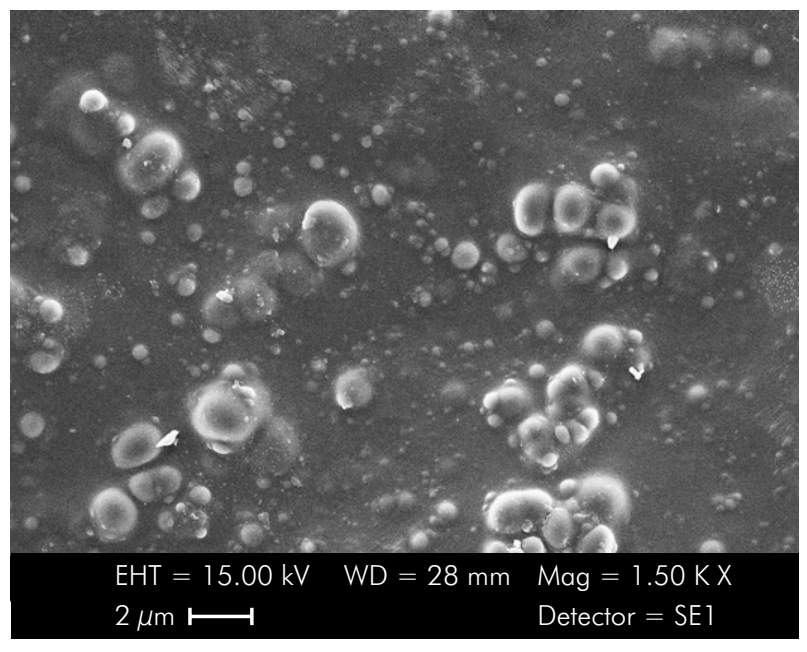

Figure 4. Scanning electron microscopy image of buccal film Z4 impregnated with zolpidem-loaded nanospheres. calculated from the concentration-time curve using a noncompartmental pharmacokinetic model. The plasma concentration versus time profile of zolpidem following the application of nanosphere-impregnated buccal films and oral administration is depicted in Figure 5; the plasma profiles of these two routes of administration were evidently different. Zolpidem was detected in the plasma within the first hour of administration via both buccal and oral routes, though the level of drug present differed. When administered through the buccal mucosa, plasma drug levels were extended, and the $\mathrm{C}_{\max }$ was attained in $1.5 \mathrm{~h}$. Moreover, the plasma concentration at each time point was found to be higher with the buccal route compared to the oral. The $\mathrm{C}_{\max }$ was $32.34 \mathrm{ng} / \mathrm{mL}$ when zolpidem was administered orally and $52.54 \mathrm{ng} / \mathrm{mL}$ when administered via nanosphere-impregnated buccal film. These findings suggest a higher drug concentration is attained when zolpidem is administered via a buccal route. Furthermore, the buccal $C_{\max }$ observed in the current study is comparable to a sublingual formulation of zolpidem administered in humans. ${ }^{2}$

The $T_{\max }$ was also found to be different for both administration routes. While the $\mathrm{T}_{\max }$ was $1.5 \mathrm{~h}$ by buccal administration, it was $1 \mathrm{~h}$ after oral administration. Even though the oral $\mathrm{T}_{\max }$ was less than the buccal, the plasma concentration of zolpidem via buccal administration at $1 \mathrm{~h}$ was 46.78 versus $32.34 \mathrm{ng} / \mathrm{ml}$ after oral administration. These

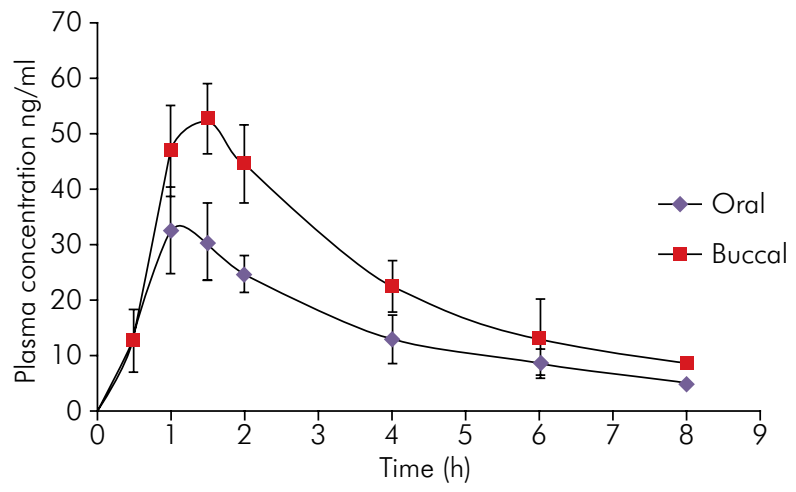

Figure 5. Comparison of plasma concentration profiles following buccal application of zolpidem nanosphere-loaded buccal film Z4 versus oral administration in rabbits. Values represent the mean of six trials \pm standard deviation. 
Table 3. Mean pharmacokinetic parameters of zolpidem in plasma following buccal application of film Z4 and oral solution in rabbits (mean $\pm S D, n=6$ ).

\begin{tabular}{lcc}
\hline Parameter & Buccal film $(\mathrm{Z} 4)$ & Oral solution \\
\hline $\mathrm{T}_{\max }(\mathrm{h})$ & 1.5 & 1 \\
$\mathrm{C}_{\max }(\mathrm{ng} / \mathrm{mL})$ & $52.54 \pm 8.22$ & $32.34 \pm 7.82$ \\
$\mathrm{AUC}_{0-\alpha}(\mathrm{ng} \cdot \mathrm{h} / \mathrm{mL})$ & $236.00 \pm 39.51^{*}$ & $136.06 \pm 28.72$ \\
\hline${ }^{*} \mathrm{p}<0.0001$ & &
\end{tabular}

results indicate the ability of the developed film to achieve a higher drug concentration in a shorter time, and hence, is purportedly a better delivery system for initiation of sleep onset. The pharmacokinetic parameters of zolpidem when administered via buccal and oral routes are listed in Table 3. It is evident that the systemic availability (area under the curve from time 0 to a) of buccal film delivery of zolpidem ( $236 \mathrm{ng} . \mathrm{h} / \mathrm{mL})$ was significantly higher than that with oral delivery ( 136.06 ng.h/mL). These findings suggest the amount of zolpidem reaching the systemic circulation following buccal administration is significantly higher $(p<0.0001)$ than via the oral route. Furthermore, the buccal route has been shown to prolong delivery of zolpidem, suggesting these films are able to maintain drug levels in the plasma for a longer period of time and may be useful for prolonging the duration of sleep. Indeed, the findings of the study are encouraging and substantiate our primary objective of designing a buccoadhesive nanosphere-impregnated drug delivery system for the delivery of zolpidem across the buccal mucosa.
The results of the current study also substantiate that the improved bioavailability of nanospheres via buccal application could be due to the transmucosal transport of zolpidem directly into the systemic circulation, compared to the oral route, which shows a relatively lower bioavailability.

\section{Conclusion}

The present study systematically evaluated the potential of nanosphere-impregnated buccoadhesive films for buccal delivery of zolpidem. Zolpidem-loaded PLGA nanospheres were prepared and loaded onto buccal films that were then evaluated using in vitro and in vivo tests. The prepared films showed adequate mucoadhesive strength and excellent physicomechanical strength. The results of in vitro dug release tests depicted the potential of the films to provide extended drug release, while ex vivo studies substantiated the potential of the nanospheres to permeate across the buccal membranes at a controlled rate. Furthermore, our in vivo studies reinforced findings from in vitro and ex vivo studies, demonstrating prolonged release and enhanced bioavailability of zolpidem.

\section{Acknowledgements}

We are grateful to Drs. Rachna Kumria and Anroop Nair for their valuable help in designing experiments and timely advice. We also acknowledge Mr. Tameem Al-Yahian (College of Clinical Pharmacy, King Faisal University, Saudi Arabia) for assistance during this study.

\section{References}

1. Ohayon MM. Epidemiology of insomnia: what we know and what we still need to learn. Sleep Med Rev. 2002;6(2):97-111. doi:10.1053/smrv.2002.0186

2. Staner L, Danjou P, Luthringer R. A new sublingual formulation of zolpidem for the treatment of sleep-onset insomnia. Expert Rev Neurother. 2012;12(2):141-53. doi:10.1586/ern.11.197

3. Schutte-Rodin S, Broch L, Buysse D, Dorsey C, Sateia M. Clinical guideline for the evaluation and management of chronic insomnia in adults. J Clin Sleep Med. 2008;4(5):487-504.

4. Basta M, Chrousos GP, Vela-Bueno A, Vgontzas AN. Chronic insomnia and stress system. Sleep Med Clin. 2007;2(2):279-91. doi:10.1016/j.jsmc.2007.04.002
5. Fiske A, Wetherell JL, Gatz M. Depression in older adults. Annu Rev Clin Psychol. 2009;5(1):363-89. doi:10.1146/annurev.clinpsy.032408.153621

6. Lautenbacher S, Kundermann B, Krieg JC. Sleep deprivation and pain perception. Sleep Med Rev. 2006;10(5):357-69. doi:10.1016/j.smrv.2005.08.001

7. Scott JP, McNaughton LR, Polman RC. Effects of sleep deprivation and exercise on cognitive, motor performance and mood. Physiol Behav. 2006;87(2):396-408. doi:10.1016/j.physbeh.2005.11.009

8. Pagel JF, Parnes BL. Medications for the treatment of sleep disorders: an overview. Prim Care Companion J Clin Psychiatry. 2001;3(3):118-25. doi:10.4088/PCC.v03n0303 
9. Rudolph U, Möhler H. GABA-based therapeutic approaches: GABAA receptor subtype functions. Curr Opin Pharmacol. 2006;6(1):18-23. doi:10.1016/j.coph.2005.10.003

10. Lieberman JA 3rd. Update on the safety considerations in the management of insomnia with hypnotics: incorporating modified-release formulations into primary care. Prim Care Companion J Clin Psychiatry. 2007;9(1):25-31. doi:10.4088/PCC.v09n0105

11. Barkin RL. Zolpidem extended-release: a single insomnia treatment option for sleep induction and sleep maintenance symptoms. Am J Ther. 2007;14(3):299-305. doi:10.1097/MJT.0b013e31804c7292

12. Moen MD, Plosker GL. Zolpidem extended-release. CNS Drugs. 2006;20(5):419-26. doi:10.2165/00023210-200620050-00006

13. Trapani G, Lopedota A, Boghetich G, Latrofa A, Franco M, Sanna E et al. Encapsulation and release of the hypnotic agent zolpidem from biodegradable polymer microparticles containing hydroxypropyl-beta-cyclodextrin. Int J Pharm. 2003;268(1-2):47-57. doi:10.1016/j.ijpharm.2003.08.016

14. Al-Dhubiab BE, Nair AB, Kumria R, Attimarad M, Harsha S. Formulation and evaluation of nano based drug delivery system for the buccal delivery of acyclovir. Colloids Surf B Biointerfaces. 2015;136:878-84. doi:10.1016/j.colsurfb.2015.10.045

15. Al-Dhubiab BE, Nair AB, Kumria R, Attimarad M, Harsha S. Development and evaluation of buccal films impregnated with selegiline-loaded nanospheres. Drug Deliv. 2015; 21:1-9. doi:10.3109/10717544.2014.948644

16. Nair AB, Kumria R, Harsha S, Attimarad M, Al-Dhubiab $\mathrm{BE}$, Alhaider IA. In vitro techniques to evaluate buccal films. J Control Release. 2013;166(1):10-21. doi:10.1016/j.jconrel.2012.11.019

17. Jacob S, Shirwaikar A, Nair A. Preparation and evaluation of fast-disintegrating effervescent tablets of glibenclamide. Drug Dev Ind Pharm. 2009;35(3):321-8. doi:10.1080/03639040802337021

18. Kumria R, Nair AB, Goomber G, Gupta S. Buccal films of prednisolone with enhanced bioavailability. Drug Deliv. 2016;23(2):471-8. doi:10.3109/10717544.2014.920058

19. Al-Dhubiab BE. Mucoadhesive buccal films embedded with antiviral drug loaded nanospheres. Turk J Pharm Sci. 2016;13(2):105-23. doi:10.5505/tjps.2016.05706

20. Al-Dhubiab BE. Formulation and In vitro evaluation of gelatin nanospheres for the oral delivery of selegiline. Curr Nanosci. 2013;9(1):21-5. doi:10.2174/1573413711309010006

21. Nair AB, Kumria R, Al-Dhubiab BE, Attimarad M, Harsha S. Noninvasive sampling of gabapentin by reverse iontophoresis. Pharm Res. 2015;32(4):1417-24. doi:10.1007/s11095-014-1546-5
22. Anroop B., Ghosh B., Parcha V., Kumar A., Khanam J. Synthesis and comparative skin permeability of atenolol and propranolol esters. J Drug Deliv Sci Technol 2005;15(2):187-90. doi:10.1016/S1773-2247(05)50025-X

23. Mudshinge SR, Deore AB, Patil S, Bhalgat CM. Nanoparticles: emerging carriers for drug delivery. Saudi Pharm J. 2011;19(3):129-41. doi:10.1016/j.jsps.2011.04.001

24. Giovino C, Ayensu I, Tetteh J, Boateng JS. Development and characterisation of chitosan films impregnated with insulin loaded PEG-b-PLA nanoparticles (NPs): a potential approach for buccal delivery of macromolecules. Int J Pharm. 2012;428(1-2):143-51. doi:10.1016/j.ijpharm.2012.02.03

25. Morales JO, Huang S, Williams RO 3rd, McConville JT. Films loaded with insulin-coated nanoparticles (ICNP) as potential platforms for peptide buccal delivery. Colloids Surf B Biointerfaces. 2014;122:38-45. doi:10.1016/j.colsurfb.2014.05.025

26. Nair A, Gupta R, Vasanti S. In vitro controlled release of alfuzosin hydrochloride using HPMC-based matrix tablets and its comparison with marketed product. Pharm Dev Technol. 2007;12(6):621-5. doi:10.1080/10837450701563277

27. Kumria R, Nair AB, Al-Dhubiab BE. Loratidine buccal films for allergic rhinitis: development and evaluation. Drug Dev Ind Pharm. 2014;40(5):625-31. doi:10.3109/03639045.2014.884125

28. Kumria R, Gupta V, Bansal S, Wadhwa J, Nair AB. Oral buccoadhesive films of ondansetron: development and evaluation. Int J Pharm Investig. 2013;3(2):112-8. doi:10.4103/2230-973X.114894

29. Nair A, Reddy C, Jacob S. Delivery of a classical antihypertensive agent through the skin by chemical enhancers and iontophoresis. Skin Res Technol. 2009;15(2):187-94. doi:10.1111/j.1600-0846.2009.00350.x

30. Anroop B, Ghosh B, Parcha V, Khanam J. Transdermal delivery of atenolol: effect of prodrugs and iontophoresis. Curr Drug Deliv. 2009;6(3):280-90. doi:10.2174/156720109788680895

31. Nair A, Vyas H, Shah J, Kumar A. Effect of permeation enhancers on the iontophoretic transport of metoprolol tartrate and the drug retention in skin. Drug Deliv. 2011;18(1):19-25. doi:10.3109/10717544.2010.509361

32. Rana P, Murthy RS. Formulation and evaluation of mucoadhesive buccal films impregnated with carvedilol nanosuspension: a potential approach for delivery of drugs having high first-pass metabolism. Drug Deliv. 2013;20(5):224-35. doi:10.3109/10717544.2013.779331

33. Nair AB, Jacob S. A simple practice guide for dose conversion between animals and human. J Basic Clin Pharm. 2016;7(2):27-31. doi:10.4103/0976-0105.177703 